\title{
The Australia Telescope campaign to study southern class I methanol masers
}

\author{
M. A.Voronkov ${ }^{1}$, K. J. Brooks ${ }^{1}$, A. M. Sobolev ${ }^{2}$, S. P. Ellingsen ${ }^{3}$, \\ A. B. Ostrovskii ${ }^{2}$ and J. L. Caswell ${ }^{1}$ \\ ${ }^{1}$ Australia Telescope National Facility, PO Box 76, Epping, NSW 1710 \\ email: Maxim.Voronkov@csiro.au \\ ${ }^{2}$ Ural State University, Lenin ave. 51, 620083 Ekaterinburg, Russia \\ ${ }^{3}$ University of Tasmania, GPO Box 252-37, Hobart, Tasmania 7000, Australia
}

\begin{abstract}
The Australia Telescope Compact Array (ATCA) and the Mopra facility have been used to search for new southern class I methanol masers at 9.9, $25(\mathrm{~J}=5)$ and $104 \mathrm{GHz}$, which are thought to trace more energetic conditions in the interface regions of molecular outflows, than the widespread class I masers at 44 and $95 \mathrm{GHz}$. One source shows a clear outflow association.
\end{abstract}

Keywords. Methanol masers, outflows

A number of surveys have been carried out to investigate various class I methanol masers and to search for an association with the other constituents of a typical massive star-forming region. The ATCA $9.9 \mathrm{GHz}$ and Mopra $104 \mathrm{GHz}$ surveys indicate that these masers are rare (three detections out of 40 targets and two detections out of 69 targets, respectively) confirming the results of the previous searches (Slysh et al. 1993; Voronkov et al. 2005). In contrast, there were 67 detections at $25 \mathrm{GHz}(\mathrm{J}=5)$ out of 102 targets observed (ATCA observations, but no accurate positions were measured for the majority of the sources), although these masers were also previously believed to be rare following a survey of Menten et al. (1986). Unlike the spectra of the $44 \mathrm{GHz}$ masers, the spectra of 9.9, 25 and $104 \mathrm{GHz}$ masers appeared to be quite simple (small number of components) and no significant correlation between the flux densities has been found. This is in line with the theoretical prediction that these masers require more energetic conditions (higher temperatures and densities) to form (Sobolev et al. 2005). Only one source, G343.12-0.06 (IRAS 16547-4247), was active in all observed transitions. Its detailed observations showed a remarkable association with a jet-driven molecular outflow (see Voronkov et al. 2006 for details). The spectra of detections are given in Fig. 1. The $25 \mathrm{GHz}$ spectra are given only for the sources with measured accurate positions. A table with the absolute positions of maser spots is available in the poster which is available from the M. Voronkov, or can be downloaded from the maser meeting website.

\section{References}

Menten, K. M., Walmsley, C. M., Henkel, C., \& Wilson, T. L. 1986, A\&̊A 157, 318

Slysh, V. I., Kalenskii, S. V., \& Val'tts, I. E. 1993, ApJ 413, L133

Sobolev, A. M., Ostrovskii, A. B., Kirsanova, M. S., Shelemei, O. V., Voronkov, M. A., \& Malyshev, A. V. 2005, in: R. Cesaroni, E. Churchwell, Walmsley M., Massive star birth: A crossroads of Astrophysics, Proc. IAU Symposium No. 227 (Cambridge: Cambridge University Press), p. 174

Voronkov, M. A., Sobolev, A. M., Ellingsen, S. P., Ostrovskii, A. B., \& Alakoz A. V. 2005, ApESS 295, 217

Voronkov, M. A., Brooks, K. J., Sobolev, A. M., Ellingsen, S. P., Ostrovskii, A. B., \& Caswell, J. L. 2006, MNRAS 373, 411 

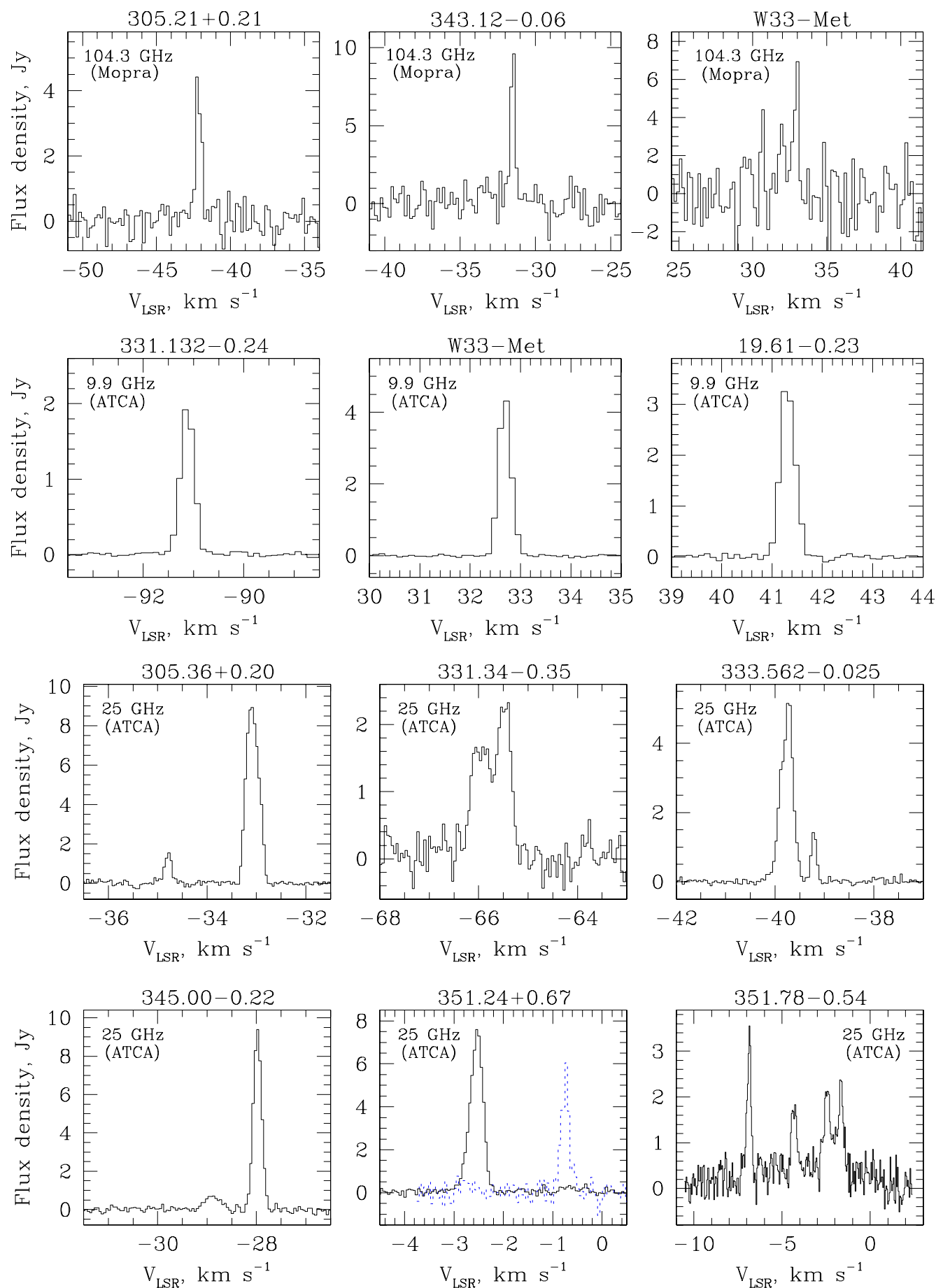

Figure 1. The spectra of the $104 \mathrm{GHz}$ (top row), $9.9 \mathrm{GHz}$ (second row) and $25 \mathrm{GHz}$ (two bottom rows) maser detections. The $25 \mathrm{GHz}$ spectra are given only for the sources with measured accurate positions. The dotted (blue) spectrum for G351.24+0.67 corresponds to an offset component (see the poster referred to in the text). 